PROCEEDINGS OF THE

AMERICAN MATHEMATICAL SOCIETY

Volume 140, Number 7, July 2012, Pages 2227-2233

S 0002-9939(2011)11086-4

Article electronically published on October 28, 2011

\title{
COHEN-KAPLANSKY DOMAINS AND THE GOLDBACH CONJECTURE
}

\author{
JIM COYKENDALL AND CHRIS SPICER
}

(Communicated by Irena Peeva)

\begin{abstract}
A Cohen-Kaplansky domain is an atomic domain with only a finite number of irreducibles. In this paper, we show that localizations of certain orders of rings of integers are necessarily CK-domains, and then prove there exists a closed form formula for the number of irreducible elements in several different cases of these types of rings. Modulo a variant of the Goldbach Conjecture, this construction allows us to answer a question posed by Cohen and Kaplansky over 60 years ago regarding the construction of a CK-domain containing $n$ nonprime irreducible elements for every positive integer $n$.
\end{abstract}

\section{INTRODUCTION AND BACKGROUND}

In 1946, Cohen and Kaplansky introduced the class of domains now known as Cohen-Kaplansky domains (CK-domains) 3]. Recall that an integral domain is atomic if every nonzero nonunit can be written as a finite product of irreducible elements. A Cohen-Kaplansky domain is an atomic domain that contains only finitely many irreducibles up to associates. As an extension of the above definition, we define a CK- $n$ domain to be a CK-domain containing precisely $n$ irreducible elements and we define a $\mathrm{CK}^{*}-n$ domain to be a CK-domain where every irreducible element is a nonprime. Implicit in their seminal work was the question as to the existence of $\mathrm{CK}^{*}-n$ domains for every positive integer $n$. This question was mentioned explicitly by D. D. Anderson in [1. Notice it is easy to construct a CK- $n$ domain for every $n$ simply by localizing $\mathbb{Z}$ at a set of $n$ distinct primes. In 2], D. D. Anderson and J. L. Mott looked at the infinite collection of D+M constructions of the form $R=k+x^{m} K[[x]]$ with $k \subseteq K$ finite fields, $m \in \mathbb{N}$. For these constructions, the closed form formula for determining the number of irreducibles is given by $n=m\left|\frac{K^{*}}{k^{*}}\right||K|^{m-1}$. This produces many examples of $\mathrm{CK}^{*}-n$ domains for small values of $n$. For instance, for values of $n<250$, this construction produces $\mathrm{CK}^{*}-n$ domains for over 100 distinct values of $n$. The smallest value of $n$ that cannot be constructed is $n=11$. The exponential nature of this formula makes it clear that the density of the output decreases with larger values of $n$.

Received by the editors October 11, 2010 and, in revised form, February 16, 2011.

2010 Mathematics Subject Classification. Primary 13F15.

(C)2011 American Mathematical Society Reverts to public domain 28 years from publication 2227 
In Section 2, we show that certain localizations of orders in a ring of integers will always be CK-domains. In particular, we utilize orders formed using inert and ramified primes and include an example as to why split primes are avoided. In Section 3, under the hypothesis of a variant of the Goldbach Conjecture, we illustrate a method that allows us to construct a $\mathrm{CK}^{*}-n$ domain for every positive integer $n \geq 6$ (and hence $n \geq 3$ in totality).

For the remainder of this paper, for an integral domain $R, K$ will always denote its quotient field and $\bar{R}$ the integral closure of $R$ in $K$. $\operatorname{Max}(R)$ is the set of maximal ideals of $R$ and $|\operatorname{Max}(R)|$ its cardinality. Also, $U(R)$ will denote the multiplicative group of units of $R$. Finally, if $X \subset R$, we define $X^{c}$ to be the set complement of $X$ in $R$.

\section{CK-DOMAins FROM LOCALiZATions}

We begin this section with some basic definitions from number theory. Any unexplained material may be found in 4 . Let $\omega$ be an element that is algebraic over $\mathbb{Q}$, and recall that $R=\{k \in \mathbb{Q}(\omega) \mid k$ is a root of a monic polynomial in $\mathbb{Z}[x]\}$ is called a ring of integers. Given a ring of integers $R$ with quotient field $K$, then an order is a subring of $R$ whose quotient field is also $K$. The main result of this section is Corollary 2.2, which gives conditions for certain localizations of these orders to be CK-domains.

To prove that a domain $R$ is a CK-domain, we will make use of the following theorem found in [2].

Theorem 2.1. For an integral domain $R$, the following conditions are equivalent.

(1) $R$ is a CK-domain.

(2) $R$ is a one-dimensional semilocal domain and for each nonprincipal maximal ideal $M$ of $R, R / M$ is finite and $R_{M}$ is analytically irreducible.

(3) $R$ is a one-dimensional semilocal domain with $R / M$ finite for each nonprincipal maximal ideal $M$ of $R, \bar{R}$ is a finitely generated $R$-module (equivalently, $(R: \bar{R}) \neq 0)$, and $|\operatorname{Max}(R)|=|\operatorname{Max}(\bar{R})|$.

(4) $\bar{R}$ is a semilocal PID, $|\operatorname{Max}(R)|=|\operatorname{Max}(\bar{R})|, \bar{R}$ is a finitely generated $R$ module, and if $M$ is a nonprincipal maximal ideal of $R$, then $R / M$ is finite.

With the above characterization in hand, we are now ready to present the main result of this section.

Corollary 2.2. Let $R \subseteq \bar{R}$ be an order in a ring of integers and for $1 \leq i \leq m$, let $p_{i}$ be distinct primes in $\mathbb{Z}$ such that each $p_{i} \bar{R}=\mathfrak{P}_{i}^{j}$ for some $j$. Define $\mathfrak{Q}_{i}=\mathfrak{P}_{i} \cap R$. If $T=\left(\mathfrak{Q}_{1} \cup \mathfrak{Q}_{2} \cup \cdots \cup \mathfrak{Q}_{m}\right)^{c}$, then $R_{T}$ is a CK-domain.

Proof. We use condition (3) from Theorem 2.1. It is well known that $R_{T}$ is a one-dimensional semilocal domain where $R_{T} / I$ is finite for every nonzero ideal of $R_{T}$. Note that $\bar{R}$ is a finitely generated $\mathbb{Z}$-module, hence a finitely generated $R$-module, and hence it is easy to see then that $\overline{R_{T}}$ is a finitely generated $R_{T^{-}}$ module. So it suffices to prove that $\left|\operatorname{Max}\left(R_{T}\right)\right|=\left|\operatorname{Max}\left(\overline{R_{T}}\right)\right|$. To this end, note that $\left|\operatorname{Max}\left(R_{T}\right)\right| \leq\left|\operatorname{Max}\left(\overline{R_{T}}\right)\right|$ since integral extensions satisfy the lying over property [4.

It will therefore suffice to show that every maximal ideal of $R_{T}$ is lain over by precisely one maximal ideal of $\overline{R_{T}}$. Suppose not. Then there must exist some $M_{1}, M_{2}, \ldots, M_{k} \in \operatorname{Max}\left(\overline{R_{T}}\right)$ such that $M_{i} \cap R_{T}=\mathfrak{Q}_{1}$ for all $1 \leq i \leq k$. Notice that 
$\mathfrak{P}_{1} \overline{R_{T}}$ must be one of the $M_{i}$, so without loss of generality, say $\mathfrak{P}_{1} \overline{R_{T}}=M_{1}$. Then $M_{i} \bar{R}_{S}=\bar{R}_{S}$ for all $i \neq 1$, where $S=\left(\mathfrak{P}_{1} \cup \cdots \cup \mathfrak{P}_{m}\right)^{c}$. If not, then some $M_{i} \bar{R}_{S} \subseteq \mathfrak{P}_{1}$ and since $M_{1}$ and $M_{i}$ are comaximal, this would imply that $\left(M_{1}, M_{i}\right)=\bar{R}_{S} \subseteq \mathfrak{P}_{1}$, which is a contradiction. Note that if $M_{i} \subseteq S^{c}=\mathfrak{P}_{1} \cup \mathfrak{P}_{2} \cup \cdots \cup \mathfrak{P}_{m}$, then $M_{i} \subseteq \mathfrak{P}_{\text {j }}$ for some $j$. Hence, $M_{i} \cap S \neq \emptyset$ for all $n \neq 1$. So let $s \in M_{i} \cap S$ and notice that since $s$ is integral over $\mathbb{Z}$, we must have $s$ being a root of some minimal polynomial, say $s^{n}+r_{n-1} s^{n-1}+\cdots+r_{1} s+r_{0}=0$ with $r_{i} \in \mathbb{Z}$. Hence $r_{0} \in M_{i} \cap \mathbb{Z}=\left(p_{1}\right)$, and $\pm r_{0}$ is the norm of $s \in\left(\mathfrak{P}_{1} \cup \mathfrak{P}_{2} \cup \cdots \cup \mathfrak{P}_{m}\right)^{c}$. However, since $p_{1} \bar{R}=\mathfrak{P}_{1}^{j}$, only elements contained in $\mathfrak{P}_{1}$ can have norm divisible by $p_{1}$. Thus it follows that $s \in \mathfrak{P}_{1}$, which is a contradiction. Thus $R$ is a CK-domain as desired.

As to why we do not consider split primes of $\mathbb{Z}$, consider the following example.

Example 2.3. First note that in $\mathbb{Z}[i]$ we have $5=(2+i)(2-i)$ a split prime. So we consider the ring $\mathbb{Z}[5 i]_{(5,5 i)}$. We claim that the set $\left\{5(2+i)^{n}\right\}_{n=1}^{\infty}$ is an infinite collection of distinct nonassociate irreducibles. The fact that these are pairwise nonassociate follows directly from the fact that each has norm $5^{n+2}$. To prove irreducibility, we induct on $n$. Note that if $n=0,1$, then $N\left(5(2+i)^{n}\right)=5^{2}$ or $5^{3}$ respectively. In $\mathbb{Z}[5 i]_{(5,5 i)}$, an arbitrary element has norm $N\left(\frac{a+5 b i}{x+5 y i}\right)=\frac{a^{2}+25 b^{2}}{x^{2}+25 y^{2}}$, where $\operatorname{gcd}(x, 5)=1$. Notice that the norm of the denominator must be relatively prime to 5 . Also, if $5 \mid\left(a^{2}+25 b^{2}\right)$, then $25 \mid\left(a^{2}+25 b^{2}\right)$. Therefore any element of either norm $5^{2}$ or $5^{3}$ must necessarily be irreducible. Now assume $5(2+i)^{n}$ is irreducible for all $0 \leq n \leq m$ and consider $5(2+i)^{m+1}$. Suppose that $5(2+i)^{m+1}=\alpha \beta$ in $\mathbb{Z}[5 i]_{(5,5 i)} \subseteq \mathbb{Z}[i]_{((2+i) \cup(2-i))^{c}}$, the larger of which is a UFD. In $\mathbb{Z}[i]_{((2+i) \cup(2-i))^{c}}$, we have, up to a unit, $5(2+i)^{m+1}=(2-i)(2+i)^{m+2}$. Therefore, either $\alpha$ or $\beta$ must be a power of $(2+i)$. Without loss of generality, write $\alpha=u(2+i)^{k}$ where $u \in U\left(\mathbb{Z}[i]_{((2+i) \cup(2-i))^{c}}\right)$ and $0<k \leq m+2$. Thus there must exist some unit $\frac{a+b i}{c+d i} \in \mathbb{Z}[i]_{((2+i) \cup(2-i))^{c}}$ such that $(2+i)^{k} \frac{a+b i}{c+d i}=\frac{x+5 y i}{u+5 v i}$ where $\operatorname{gcd}(u, 5)=1$, $c^{2}+d^{2} \not \equiv 0 \bmod 5$, and $a^{2}+b^{2} \not \equiv 0 \bmod 5$. Simplifying yields

$$
(2+i)^{k}(a+b i)(u+5 v i)=(c+d i)(x+5 y i) \in \mathbb{Z}[i]_{((2+i) \cup(2-i))^{c}},
$$

which implies $(2+i) \mid(x+5 y i)$ and hence $5 \mid x$. But this means that $(2-i) \mid(x+5 y i)$, which implies $(2-i) \mid(a+b i)$, which contradicts the fact that $a^{2}+b^{2} \not \equiv 0 \bmod 5$. Thus $5(2+i)^{m+1}$ is irreducible as desired.

\section{CK-DOMAINS WITH A SPECIFIED NUMBER OF IRREDUCIBLES}

We now turn our attention to the goal of creating a $\mathrm{CK}^{*}-n$ domain for every $n \in \mathbb{Z}^{+}$. We begin by applying Corollary 2.2 to the case of quadratic rings of integers. This allows us to explicitly create irreducibles in certain localizations.

Theorem 3.1. Let $d$ be a square-free integer and $\mathbb{Z}[\omega]$ the ring of integers of $\mathbb{Q}[\sqrt{d}]$. If $p$ is an inert prime in $\mathbb{Z}[\omega]$, then $R=\mathbb{Z}[p \omega]_{(p, p \omega)}$ is a $C K^{*}-(p+1)$ domain.

Proof. We will restrict ourselves to the case of $\omega=\sqrt{d}$, where $d \equiv 2,3 \bmod 4$. The case of $d \equiv 1 \bmod 4$ follows in a similar fashion. We first note that $(p, p \sqrt{d})$ is a prime ideal of $\mathbb{Z}[p \sqrt{d}]$ and that the integral closure of $R$ is $T=\mathbb{Z}[\sqrt{d}]_{(p)}=$ $\left\{\frac{a+b \sqrt{d}}{x+y \sqrt{d}}: a, b, x, y \in \mathbb{Z}, \operatorname{gcd}(x, p)=\operatorname{gcd}(y, p)=1\right\}$. Viewing each element of $R$ then as an element of $T$, we see that every nonzero element of $R$ can be written as some unit multiple of $p^{j}$ for some $j \in \mathbb{N}_{0}$. This follows since $T$ is a Noetherian 
valuation domain with maximal ideal generated by $p$. Therefore every nonzero element of $R$ can be written, up to a unit of $R$, in the form $p^{j}(a+b \sqrt{d})$ for some $a, b \in \mathbb{Z}$ since $R$ and $T$ are both local and there is no loss in generality in simply clearing denominators. In an effort to count the irreducible elements of $R$, we first notice that we need only consider those elements having $j=1$. To see why, notice that if we had some element, say $p^{n}(a+b \sqrt{d})$ for $n \geq 2$, then this would factor as $p\left(p^{n-1}(a+b \sqrt{d})\right)$ and hence cannot be irreducible. So it will suffice to consider the number of equivalence classes of irreducibles of the form $p(a+b \sqrt{d})$, where we declare two irreducibles to be in the same equivalence class if $p(a+b \sqrt{d})=p(x+y \sqrt{d})(u)$, with $u=\frac{\alpha+\beta p \sqrt{d}}{\gamma+\delta p \sqrt{d}}$ and $\operatorname{gcd}(\alpha, p)=\operatorname{gcd}(\gamma, p)=1$.

We first show that if $x \equiv a \bmod p$ and $y \equiv b \bmod p$, then $p(a+b \sqrt{d}) \sim p(x+y \sqrt{d})$. Write $x=a+k p$ and $y=b+l p$ for $k, l \in \mathbb{Z}^{+}$. Then $\frac{x+y \sqrt{d}}{a+b \sqrt{d}}=1+\frac{p(k+l \sqrt{d})}{a+b \sqrt{d}}=$ $1+p \frac{(k+l \sqrt{d})(a-b \sqrt{d})}{a^{2}-b^{2} d}$. Notice that since $p \frac{(k+l \sqrt{d})(a-b \sqrt{d})}{a^{2}-b^{2} d}$ is in $(p, p \sqrt{d})$, it follows that $1+p \frac{(k+l \sqrt{d})(a-b \sqrt{d})}{a^{2}-b^{2} d}$ is a unit of $R$. Thus $x+y \sqrt{d} \sim a+b \sqrt{d}$ as desired. We are now considering irreducible equivalence classes of the form $p(a+b \sqrt{d})$. Notice first that if $a \equiv 0 \bmod p$, then we obtain a single irreducible equivalence class with representative $(p \sqrt{d})$. If $a \not \equiv 0 \bmod p$, then there is no loss in generality in assuming $a \equiv 1 \bmod p$ simply by multiplying through by $a^{-1}(\bmod p)$. It will suffice to show that we obtain $p$ unique equivalence classes whose representatives can be expressed in the form $p(1+r \sqrt{d})$, where $r=0,1, \ldots, p-1$. So suppose $p(1+a \sqrt{d}) \sim p(1+b \sqrt{d})$. Then we must have $p(1+a \sqrt{d})=p(1+b \sqrt{d})\left(\frac{\alpha+\beta p \sqrt{d}}{\gamma+\delta p \sqrt{d}}\right)$ with $\operatorname{gcd}(\alpha, p)=\operatorname{gcd}(\gamma, p)=1$. Multiplying across and simplifying, we obtain $(\gamma+a d \delta p)+(a \gamma+\delta p) \sqrt{d}=(\alpha+d b \beta p)+(\beta p+\alpha b) \sqrt{d}$. When taken mod $p$, notice we must have $\gamma \equiv \alpha \bmod p$ and $a \gamma \equiv \alpha b \bmod p$. This implies that $a \alpha \equiv \alpha b \bmod p$ and hence $a \equiv b \bmod p$. Thus we have a total of $p+1$ irreducible elements corresponding to the equivalence classes of: $(p \sqrt{d}),(p), p(1+\sqrt{d}), p(1+2 \sqrt{d}), \ldots, p(1+(p-1) \sqrt{d})$, and hence $R$ is a $\mathrm{CK}^{*}-(p+1)$ domain as claimed.

This yields the following immediate corollary.

Corollary 3.2. Let $R=\mathbb{Z}[p n \omega]_{(p, p n \omega)}$, where $p$ is an inert prime in $\mathbb{Z}[\omega]$ and $\operatorname{gcd}(p, n)=1$. Then $R$ is also a $C K^{*}-(p+1)$ domain.

Proof. We need only consider those equivalence classes of irreducibles of the form $p(a+b n \sqrt{d})$. Since $\operatorname{gcd}(p, n)=1$, the computations follow the proof of Theorem 3.1. and we thus obtain the irreducible equivalence classes with representatives $(p), p(1+r n \sqrt{d})$ for $r=0, \ldots, p-1$.

The above theorem and corollary now allow us to create a $\mathrm{CK}^{*}-n$ domain for every integer $n$ that can be expressed as $p+1$ for some prime $p \in \mathbb{Z}$. However, for the remaining $n$, we will need to localize at more than one maximal ideal. We will rely on the following lemmata found in $[3$.

Lemma 3.3. Two maximal ideals of the CK-domain $R$ cannot have an irreducible element in common. 
Lemma 3.4. Let $\mathfrak{M}$ be a maximal ideal of the $C K$-domain $R$. Then the irreducible elements of $R_{\mathfrak{M}}$ are precisely the irreducible elements of $R$ which lie in $\mathfrak{M}$.

As a first step in the application of the above lemma, we consider the case of two maximal ideals below.

Theorem 3.5. Let $S=((p, p q \omega) \cup(q, p q \omega))^{c}$ and let $R=\mathbb{Z}[p q \omega]_{S}$, where $p, q$ are distinct inert primes in $\mathbb{Z}[\omega]$. Then $R$ is a $C K^{*}-((p+1)+(q+1))$ domain.

Proof. First note that by Corollary 3.2 we have that $\mathbb{Z}[p q \omega]_{(p, p q \omega)}$ is a $\mathrm{CK}^{*}-(p+1)$ domain and that by symmetry, $\mathbb{Z}[p q \omega]_{(q, p q \omega)}$ must be a $\mathrm{CK}^{*}-(q+1)$ domain. Notice also that $R$ is a domain containing precisely two maximal ideals, namely $(p, p q \omega)$ and $(q, p q \omega)$. It is clear then from Lemma 3.4 that if $R$ is a CK $^{*}$-domain, we must have that $R$ has precisely $(p+1)+(q+1)$ irreducible equivalence classes. So it will suffice to show that $R$ is a $\mathrm{CK}^{*}$-domain. First notice that, as before, there is an immediate upper bound on the size of possible powers of $p$ and $q$ to consider. That is, we need only be concerned with equivalence classes of the form $p(a+b q \omega)$ or $q(x+y p \omega)$. Any equivalence class having greater powers of $p$ or $q$ must then necessarily reduce since we can always factor as $p^{n-1}[p(a+b q \omega)]$. In a similar manner, we need not concern ourselves with elements of the form $p q(a+b \omega)$ since they can always be expressed in the form of $p(q a+q b \omega)$ or $q(p a+p b \omega)$. Again, we will show that $\omega=\sqrt{d}$, where $d \equiv 2,3 \bmod 4$, and leave the case of $d \equiv 1 \bmod 4$ to the reader.

By an identical argument as in Theorem 3.1, if $x \equiv a \bmod p$ and $y \equiv b \bmod p$, then let $p(a+b q \sqrt{d}) \sim p(x+y q \sqrt{d})$. So, by symmetry, it will suffice to count the number of irreducibles of the form $p(a+b q \sqrt{d})$. The only distinction between this case and Theorem 3.1 is the case of $a \equiv 0 \bmod p$. Before, we chose the representative $(p q \sqrt{d})$. However, we will see from Lemma 3.3 that the element $p q \sqrt{d}$ cannot be irreducible since it lies in both maximal ideals $(p, p q \sqrt{d})$ and $(q, q p \sqrt{d})$. Thus an appropriate equivalence class representative for the case of $a \equiv 0 \bmod p$ would instead be $p(p+q \sqrt{d})$. If $a \neq 10 \bmod p$, then, as in Theorem 3.1, there is no loss in generality in assuming $a \equiv 1 \bmod p$. We then obtain the remaining $p$ irreducibles, which can be expressed as unit multiples of the form $p(1+r q \sqrt{d})$ for $r=0, \ldots, p-1$. The proof that these are distinct directly follows the proof of the similar fact in Theorem 3.1. Thus, by symmetry, we also obtain the $q+1$ irreducible equivalence classes corresponding to associates of the form $q(q+p \sqrt{d})$ and $q(1+r p \sqrt{d})$. Hence $R$ is a CK$^{*}-((p+1)+(q+1))$ domain as desired.

The fact that we considered only two distinct inert primes was arbitrary. Thus we have the following immediate corollary.

Corollary 3.6. Let $S=\left(\left(p_{1}, p \omega\right) \cup\left(p_{2}, p \omega\right) \cup \cdots \cup\left(p_{n}, p \omega\right)\right)^{c}$, where $p=p_{1} \cdots p_{n}$, and $p_{1}, p_{2}, \ldots, p_{n}$ are distinct inert primes in $\mathbb{Z}[\omega]$, and let $R=\mathbb{Z}[p \omega]_{S}$. Then $R$ is a $C K^{*}$-domain containing $\sum_{i=1}^{n}\left(p_{i}+1\right)=\left(\sum_{i=1}^{n} p_{i}\right)+n$ distinct irreducible elements.

With the above theorems in hand, we are now ready to construct a $\mathrm{CK}^{*}$-domain with a precise number of irreducibles. In order to do this, we will have to make use of the Goldbach Conjecture, which simply states that every even integer greater than 2 can be expressed as the sum of two primes. However, the construction below will need the additional hypothesis that the two primes be distinct. 
Conjecture 3.7. Every even integer greater than six can be expressed as the sum of two distinct primes.

Clearly this modified conjecture implies the Goldbach Conjecture. The authors are unsure as to the status of the equivalence. However, assuming the Goldbach Conjecture, the only potential hazards are even integers of the form $2 p$ for $p$ a prime. It has been empirically verified for the first 10,000 primes that the conjectures are equivalent.

Theorem 3.8. If every even integer greater than six can be expressed as the sum of two distinct primes, then there exists a $C K^{*}-n$ domain for every positive integer $n>3$.

Proof. Let $n$ be an arbitrary positive integer. The power series construction described in [2] allows one to construct $\mathrm{CK}^{*}$-domains for every $n<11$, so we only consider the case where $n \geq 11$. The case of $n=11$ will be discussed last. This allows us to apply the modified Goldbach Conjecture. If $n$ is even, then $n-2$ is even and by the modified Goldbach Conjecture, $n-2=p+q$ for some distinct primes $p$ and $q$. From [4], we know that there must exist some quadratic ring of integers, say without loss of generality $\mathbb{Z}[\omega]$, where $p$ and $q$ are inert primes. Then $R=\mathbb{Z}[p q \omega]_{((p, p q \omega) \cup(q, p q \omega))^{c}}$ is a $\mathrm{CK}^{*}$-domain containing $(p+1)+(q+1)=n$ distinct irreducibles. For any odd integer $n=2 k+1$ for some $k>0$, first note that if we can find a quadratic ring of integers where 2 is an inert prime, then we can construct a CK* ${ }^{*} 3$ domain by localizing at the maximal ideal lying over 2 . So consider $n-5=2 k-4$, which is an even integer. By the modified Goldbach Conjecture, we must have $2 k-4=p+q$, where $p$ and $q$ are distinct odd primes. From [4, we know there exists a quadratic ring of integers such that $2, p$ and $q$ are all inert primes, say $\mathbb{Z}[\omega]$. Then $\mathbb{Z}[2 p q \omega]_{((2,2 p q \omega) \cup(p, 2 p q \omega) \cup(q, 2 p q \omega))^{c}}$ is a $\mathrm{CK}^{*}$-domain and, by Corollary [3.6. contains $(2+1)+(p+1)+(q+1)=5+(2 k-4)=2 k+1=n$ irreducible elements as desired. For the case of $n=11$, we mimic the odd case, only now we use the primes 2 and 7 . For an explicit quadratic ring of integers, consider $\mathbb{Z}\left[\left(\frac{1+\sqrt{5}}{2}\right)\right]=\mathbb{Z}[\omega]$. Then $\mathbb{Z}[2 \cdot 7 \omega]_{((2,2 \cdot 7 \omega)) \cup(7,2 \cdot 7 \omega))^{c}}$ is a $\mathrm{CK}^{*}-11$ domain.

We remark that this quadratic localization construction allows us to construct a $\mathrm{CK}^{*}-n$ domain for every $n \geq 3$ except for 5 . It is an interesting question, originally posed in [3], to consider for some positive integer $n$ the number of distinct multiplicative structures that can occur in a $\mathrm{CK}^{*}-n$ domain. As a refinement to this question, we define CK- $n, m$ to be a CK- $n$ domain with $1 \leq m \leq n$ maximal ideals. Note that, for example, a CK- $n, n$ domain will always be a UFD. One might ask how many distinct types of CK-domains occur for a fixed $n, m$. Also, given an $n$, what values of $m$ can occur in a CK-domain?

\section{REFERENCES}

1. D. D. Anderson, Some Finiteness Conditions on a Commutative Ring, Houston J. Math., 4, No. 3 (1978), 289-299. MR.514243 (80c:13013)

2. D. D. Anderson and J. L. Mott, Cohen-Kaplansky Domains: Integral Domains with a Finite Number of Irreducible Elements, J. Algebra, 148 (1992), 17-41. MR1161563 (93e:13041) 
3. I.S. Cohen and I. Kaplansky, Rings with a Finite Number of Primes, Trans. Amer. Math. Soc., 60 (1946), 468-477. MR0019595 (8:434a)

4. R. Gilmer, Multiplicative Ideal Theory, Queen's Papers in Pure and Appl. Math., vol. 90, Kingston, 1992, 513-516. MR:1204267 (93j:13001)

Department of Mathematics, North Dakota State University, Fargo, North Dakota 58108-6050

E-mail address: jim.coykendall@ndsu.edu

Department of Mathematical Sciences, Morningside College, Sioux City, Iowa 511061717

E-mail address: spicer@morningside.edu 